\title{
Can sexual selection drive female life histories? A comparative study on Galliform birds
}

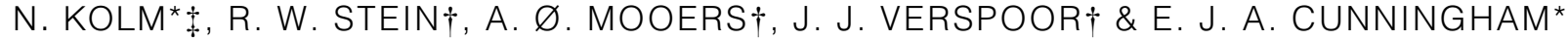 \\ *Institute of Evolutionary Biology, School of Biological Sciences, Ashworth Laboratories, University of Edinburgh, Edinburgh, Scotland, UK \\ $\dagger$ Department of Biological Sciences, Simon Fraser University, Burnaby, BC, Canada \\ $\$$ Animal Ecology/Department of Ecology and Evolution, Evolutionary Biology Centre, Uppsala University, Norbyvagen, Uppsala, Sweden
}

\section{Keywords:}

body size;

clutch size;

DISCRETE;

egg size;

mating system;

sexual size dimorphism;

supertree phylogeny;

trade-off.

\begin{abstract}
Sexual selection has been identified as a major evolutionary force shaping male life history traits but its impact on female life history evolution is less clear. Here we examine the impact of sexual selection on three key female traits (body size, egg size and clutch size) in Galliform birds. Using comparative independent contrast analyses and directional Discrete analyses, based on published data and a new genera-level supertree phylogeny of Galliform birds, we investigated how sexual selection [quantified as sexual size dimorphism (SSD) and social mating system (MS)] affects these three important female traits. We found that female body mass was strongly and positively correlated with egg size but not with clutch size, and that clutch size decreased as egg size increased. We established that SSD was related to MS, and then used SSD as a proxy of the strength of sexual selection. We found both a positive relationship between SSD and female body mass and egg size and that increases in female body mass and egg size tend to occur following increases in SSD in this bird order. This pattern of female body mass increases lagging behind changes in SSD, established using our directional DISCRETE analysis, suggests that female body mass increases as a response to increases in the level of sexual selection and not simply through a strong genetic relationship with male body mass. This suggests that sexual selection is linked to changes in female life history traits in Galliformes and we discuss how this link may shape patterns of life history variation among species.
\end{abstract}

\section{Introduction}

Sexual selection is an important driver of many of the most spectacular morphological traits that we find in the animal kingdom (for example see Andersson, 1994). As such, sexual selection is most often emphasized as a driver of the evolution of male-specific traits because sexual selection often acts more strongly on males (Andersson, 1994). However, recent studies have shown that sexual selection on male characters also can influence female life history traits, both directly (e.g. Cunningham \& Russell, 2000; Sheldon, 2000; Kolm, 2001) and indirectly, potentially through genetic correlations

Correspondence: Niclas Kolm, Institute of Evolutionary Biology, School of Biological Sciences, Ashworth Laboratories, University of Edinburgh, Edinburgh EH9 3JT, Scotland, UK.

Tel: +46 184 712639; fax: +46 184716484 ;

e-mail: niclas.kolm@ebc.uu.se for traits among the sexes (e.g. Weatherhead $\&$ Teather, 1994; Young, 2005). Here we focus on three female traits which are tightly related to fitness in a range of taxa: body mass, egg size and clutch size (e.g. Roff, 1992; Stearns, 1992; Heath \& Blouw, 1998; Roff, 2002). Recent studies have suggested that these traits are particularly strong candidates for being influenced by sexual selection (review by Sheldon, 2000; Kolm, 2001; review by Kolm \& Ahnesjö, 2005).

Birds have been studied extensively in relation to both the evolution of life histories and sexual selection. As birds show high variation in these traits, they are well suited for analyses looking at broad scale evolutionary patterns. This has been done both across major lineages of birds (e.g. Bennett $\&$ Owens, 2002) as well as on a finer, family, genus or species level scale (e.g. Blackburn, 1991a; Weatherhead \& Teather, 1994; Lindenfors et al., 2003; Figuerola \& Green, 2006; Martin et al., 2006); 
however, the evolutionary relationships between body size, egg size and clutch size, and the relationships of these with sexual selection, remain unclear. Although most studies agree that female size is positively related to egg size (Bennett 8 Owens, 2002 and references therein, Martin et al., 2006), the relationship to clutch size varies: Bennett \& Owens (2002) found no relationship between body size and clutch size across major lineages of birds whereas Martin et al. (2006) recently found a negative relationship between body size and clutch size among passerines. Further, although some studies have found a negative relationship between egg size and clutch size (Blackburn, 199la,b; Figuerola \& Green, 2006; Martin et al., 2006) indicating a trade-off between the two traits, other studies have failed to detect such a relationship (e.g. Saether, 1987; Rohwer, 1988; Poiani \& Jermiin, 1994; Bennett \& Owens, 2002).

The two most common measures of sexual selection are sexual size dimorphism (SSD) and social mating system (MS) (Bennett \& Owens, 2002). The most common type of SSD, where the male is larger than the female, is believed to be generated under sexual selection because of selection for increased male size, or more rarely because of a decrease in female size (Andersson, 1994). As polygynous species tend to involve more malemale competition [which selects for increased male size (Andersson, 1994)] when compared with monogamous species, it is not surprising that level of polygyny and SSD are related (e.g. Owens \& Hartley, 1998; Dunn et al., 2001, Bennett \& Owens, 2002). Regarding the link between measures of sexual selection and life histories, Weatherhead \& Teather (1994) found that egg size increased with increased levels of SSD across six groups of birds and Figuerola \& Green (2006) recently found the same pattern in Anseriformes. However, Bennett $\delta$ Owens' (2002) larger scale analyses across major lineages of birds did not detect any relationship between sexual selection and female life history traits.

Here we examine the impact of sexual selection on female size, egg size and clutch size among Galliform birds, the group from which all our commercially important poultry and game birds are derived. Importantly here, Galliform birds produce precocial young and hence any trade-off between egg size and egg number is less likely to be confounded by the later evolution of extensive parental care strategies which can shift investment from egg allocation to parental allocation stages. Moreover, Galliform birds show a substantial variation in life history traits as well as levels of sexual selection and are therefore well-suited for comparative analyses on the link between sexual selection and life histories.

Comparative analyses that control for shared ancestry between species are often used in order to investigate covariation among different life history traits in evolutionary biology (see e.g. Harvey \& Pagel, 1991). Together with the analysis of independent contrast (Felsenstein, 1985), the Discrete method (e.g. Pagel, 1994, 1997, 1999), based on a simple Markov model for trait evolution, is particularly appealing for these types of studies because it allows for analyses of directionality of evolution among correlated traits. This means that it is possible not only to investigate if traits evolve in relation to other traits but also to investigate if changes in one trait tend to precede changes in another trait and so to order the events involved in correlated evolution. Different orderings may support different causal explanations. Discrete has been successfully used to disentangle the association among various traits in different taxa (e.g. birds: coloniality, territoriality and habitat, Rolland et al., 1998; mate fidelity and site fidelity, Cézilly et al., 2000; breeding strategy, breeding range, diet and egg size, Krüger $\delta$ Davies, 2002; fish: body size, egg size and clutch size, Kolm et al., 2006). Here, we use this method to disentangle the direction of events in scenarios where sexual selection may drive the evolution of life histories.

To investigate this in Galliformes, we (R. W. S., J. J. V. $\delta$ A. Ø. M.) assembled a new genus-level supertree for Galliformes and we established a database of life history traits and various measures of sexual selection for the 79 genera from this group. The complex associations between different life history traits in birds make it important to investigate the link between life history traits prior to investigating any link between sexual selection and life histories. Hence, we first investigated the relationships between female size, egg size and clutch size using both analyses of raw data and phylogenetically independent contrasts (PICs). We then examined if MS was related to the degree of SSD and how SSD was linked to these female traits using both correlation analyses (based on raw data and contrasts) as well as directional DISCRETE analyses.

\section{Methods}

\section{Database}

We constructed a database for 82-214 species (32-63 genera) for which we could find information pertaining to any of the characters of interest: these included male body weight (grams: 180 species from 60 genera), female body weight (grams: 164 species from 53 genera), clutch size (number of eggs in clutch: 214 species from 63 genera), egg size $\left(\mathrm{cm}^{3}: 74\right.$ species from 40 genera) and social MS (82 species from 32 genera). These data originated from Cramp \& Simmons (1980); Dunning (1993); del Hoyo et al. (1994) and Geffen \& Yom-Tov (2001). The data for the variables under investigation were calculated using the average of the species for which we had data and all analyses were performed at the genus level. Body size was quantified as body mass. Egg size was quantified as total egg volume but sometimes, when only measures of egg length and width could be obtained from the literature, we used the Hoyt (1979) egg volume equation with the constant set to 
0.5025 [average constant over Galliform genera given in Hoyt (1979)] to calculate egg volume. Clutch size was estimated as number of eggs laid per reproductive event. SSD was calculated using the common $\log _{10}$ (male weight/female weight) calculation (e.g. Fairbairn, 1997; Young, 2005). For a genus where males are larger than females this yields a positive value for this measure of SSD. For MS, we used a majority rule and scored a genus according to the majority of the species for which we had access to data. For simplicity, we only scored genera as monogamous or polygamous. This means that genera normally considered promiscuous will fall into the polygamous category in our analyses. As we only use MS as a measure of sexual selection, this procedure should not introduce any biases. The only genus recognized as polyandrous (genus Alectura) was removed from all analyses of MS to ensure a binary state for this variable. For the Discrete analyses, which require binary coded characters, we transformed the continuous variable SSD into a binary variable by scoring all genera above the mean (across all genera) as 1 and all genera below the mean (across all genera) as 0. For female body mass, egg size and clutch size we scored all genera above the mean as 1 and all genera below the mean as 0 .

\section{Supertree phylogeny}

We collected 40 previously published or 'in press' studies from a variety of sources that reported 72 phylogenetic hypotheses, hereafter referred to as source trees (STs), for genera-level matrix representation with parsimony (MRP) supertree analyses. These studies were screened for data duplication and quality prior to a more rigorous evaluation of their contained STs (see below). Three of these studies were excluded because of complete data duplication with subsequent, more inclusive studies by the same authors. In all instances, we preferred more recent molecular studies using cladistic methodologies (deemed higher quality) to older morphometric studies using either clustering algorithms or no formal analysis; this resulted in the exclusion of three additional studies. With one exception (see below), we limited our selection to studies published after 1966.

We used the 'garbage in, garbage out' protocol of Bininda-Emonds et al. (2004) as our criteria for ST selection. We selected the most comprehensive ST presented in each study; the only exceptions to this occurred when multiple STs were reported and there was data duplication involving the most comprehensive ST. Independence among STs was assessed conservatively, STs were excluded on the basis of relatively minor data duplication among studies. We identified 17 independent STs (Holman, 1961; Crowe, 1978; Gutiérrez et al., 1983; Helm-Bychowski \& Wilson, 1986; Sibley \& Ahlquist, 1990; Randi et al., 1991; Zink \& Blackwell, 1998; Armstrong et al., 2001; Birks \& Edwards, 2002; Dimcheff et al., 2002; Drovetski, 2002; Pereira et al., 2002; Sorenson et al., 2003;
Chubb, 2004; Nishibori et al., 2004; Pereira \& Baker, 2004; Crowe et al., 2006). In addition, we also identified two nucleotide sequences, mitochondrial control region and cytochrome $b$, that were recycled extensively across a further 16 studies; this resulted in two sets of nonindependent STs, one for each of these mitochondrial sequences. For these two sets, we followed the recommendation of Bininda-Emonds et al. (2004), and conducted an interim 'mini supertree' analysis on all of the available STs (control region: Fumihito et al., 1995; Kimball et al., 1997, 1999; Lucchini et al., 2001; Drovetski, 2002; and cytochrome b: Kornegay et al., 1993; Ellsworth et al., 1996; Kimball et al., 1997, 1999, 2001; Bloomer \& Crowe, 1998; Munechika et al., 1999; Gutiérrez et al., 2000; Armstrong et al., 2001; Bush \& Strobeck, 2003; Zhan et al., 2003; Shibusawa et al., 2004; Wen et al., 2005) and included the resulting 'mini supertrees' as STs in the main supertree analyses. Because of insufficient overlap among taxa, there were four, rather than two, resulting 'mini supertrees'. In an attempt to balance the quality of the included STs with taxonomic coverage, we included one osteological taxonomy of the Odontophoridae (Holman, 1961); it was highly congruent with two less complete STs from molecular studies that address relationships among genera (Zink \& Blackwell, 1998) and also with other families (Gutiérrez et al., 1983). Prior to coding the STs for MRP, nodes with published bootstrap support values $<50 \%$ were collapsed.

Wilkinson et al. (2005) recently compared the properties of 14 supertree methods and demonstrated systematic biases in the way conflicts are resolved among STs: binary coding tends to resolve conflicts in favour of unbalanced STs, whereas additive binary coding tends to resolve conflicts in favour of balanced STs (Wilkinson et al., 2005). We therefore used both coding methods [in RADCON (Thorely \& Page, 2000)] to generate matrix representation of STs: binary coding (Baum, 1992; Ragan, 1992) and Purvis' modification of this method (additive binary coding; Purvis, 1995), which eliminates redundancy inherent to binary coding (Purvis, 1995). Our MRP 'mini supertree' and main supertree analyses were conducted using the parsimony ratchet (Nixon, 1999), which increases the efficiency of heuristic searches for candidate trees, as implemented by PAUPRAT (Sikes \& Lewis, 2001) in PAuP* (Swofford, 2002). STs were weighted uniformly, that is to say that the initial weight of all characters was set to 1 , which is the default setting of PAUPRAT (Sikes $\&$ Lewis, 2001). For each of the MRP matrices we ran 30 independent searches consisting of 200 iterations, with $15 \%$ of the characters perturbed at each iteration. After the 30 independent searches we extracted the set of optimal candidate trees (shortest length) and removed duplicates trees. The ratchet searches returned 893 and 338 unique optimal candidate trees for the binary coded and the additive binary coded STs respectively. We generated the $50 \%$ majority rule 
consensus supertrees from these two sets of unique optimal candidate trees in PAUP*.

The $50 \%$ majority rule consensus supertrees from the two coding methods were highly congruent and both contained a large polytomy associated with the most recent radiation, the Phasianidae. Although Numididae was consistently placed as sister to Odontophoridae and Phasianidae in both $50 \%$ majority rule consensus supertrees, the support for this node was relatively weak (binary coding: 58\% and additive binary coding: 54\%). To account for this family-level uncertainty, we resolved both candidate supertrees (binary and additive binary) in each of two ways: with Odontophoridae as sister to Numididae and Phasianidae, and with Numididae as sister to Odontophoridae and Phasianidae. Although highly congruent, minor discrepancies did exist between the supertree topologies. So, we consulted the underlying STs, and, in all instances, the supertree constructed from additive binary coded STs matched the STs better. Because of the lack of redundancy in coding, additive binary coding is arguably a better representation of the STs than binary coding (Purvis, 1995). We performed the comparative analyses on both candidate supertrees and the results did not differ, so we only present results from analyses based on the supertree constructed from additive binary coded STs.

We attempted to provide resolution to the polytomy encompassing the Phasianidae, as follows. First, we assumed monophyly for each unambiguous branch of the polytomy, and resolved discrepancies between the supertree topologies conservatively. As a result, Alectoris, Pternistis, Rollulus, (Xenoperdix with Arborophila), and (Coturnix with Margaroperdix) were each considered as additional branches. Because of inconsistent or ambiguous affinities among the STs, Meleagris, Perdix, Tragopan, (Afropavo with Pavo), and (Rheinardia with Argusianus) were also considered as separate monophyletic branches. This increased the size of the polytomy to 18 branches. We then obtained sequence data from GenBank for six genes or introns: three mitochondrial (cytochrome $b$, ND2 and 12S rDNA) and three nuclear (ovomucoid intron G, WPG pseudogene and zona pellucida C). For each sequence, we generated a consensus sequence for each of the 18 branches; this facilitated a more thorough search of tree space at the level of the polytomy. We made a global alignment for all of the sequences for each gene in kPrank (Higgins et al., 2005; Loytynoja \& Goldman, 2005), using a guide tree imported from ClustalX (Thompson et al., 1997). From this global alignment, we then generated a consensus sequences for each branch of the polytomy. Preliminary work revealed that an $80 \%$ threshold for representation in the consensus sequence resulted in $>99 \%$ identity to ancestral sequences inferred using Bayesian methods (results not shown). Ambiguous sites in the consensus sequences, i.e. when no single nucleotide met the $80 \%$ threshold, were represented by the corresponding IUB
DNA symbol (Cornishbowden, 1986). We used MRMODELtest 2.2 (Nylander, 2004) to determine the best model of nucleotide evolution for each partition. Based on a concatenated partitioned alignment of all six sequences and with Numida meleagris as the outgroup, we inferred a phylogeny for these 18 clades using MRBAyES 3.1 .2 (Huelsenbeck \& Ronquist, 200 1; Ronquist \& Huelsenbeck, 2003). We ran four chains simultaneously in a Metropoliscoupled MCMC search of tree space in two independent iterations of 10 million generations, using default settings. After a burn in of 2.5 million generations, we sampled trees every 1000 generations; this resulted in 7500 candidate trees from which we constructed a $50 \%$ majority rule consensus.

We grafted the resulting genera-level phylogeny of the Phasianidae onto the supertree constructed from the additive binary coded STs (Fig. 1c), producing a composite supertree phylogeny (Fig. lb). From the composite supertree phylogeny, we also generated a fully resolved supertree topology based on our 'best informed guess' (BIG) of the remaining unresolved relationships (Fig. la). Finally, we allowed for the same family-level uncertainty in the relationships among Numididae, Odontophoridae and Phasianidae, resolving both the composite and BIG phylogenies in each of the two possible ways.

\section{Analyses}

Bivariate contrast analyses on the relationships between all variables were performed both using raw data and Model 1 regression through the origin using PICs (Felsenstein, 1985). For the analysis of the relationship between egg size and clutch size we also controlled for female body mass by including all three variables in a multiple regression including the 33 genera for which we had data for all three variables. All branch lengths were set equal to one for the PIC analyses and polytomies were resolved to zero-length branch lengths for the analyses based on the consensus trees (the BIG trees were fully resolved). We then tested for correlations between contrasts and their SD to check whether branch length transformations were needed to avoid type I error (Diaz-Uriarte \& Garland, 1998). As we did not detect any relationships between absolute values for the contrasts and their SD for any of the analyses, no transformations were needed. As our supertree analyses generated eight different trees [two fully resolved BIG trees based on the additive binary coded supertree (Fig. la), two composite super trees with the additive binary supertree (Fig. 1b), two supertrees based on additive binary coding (Fig. 1c) and two supertrees based on binary coding (not presented here)], we performed all analyses on all eight trees to investigate if our analyses were sensitive to which tree we used. As the results were similar (i.e. no results changed from significance to nonsignificance or vice versa) with only two exceptions 
(a)

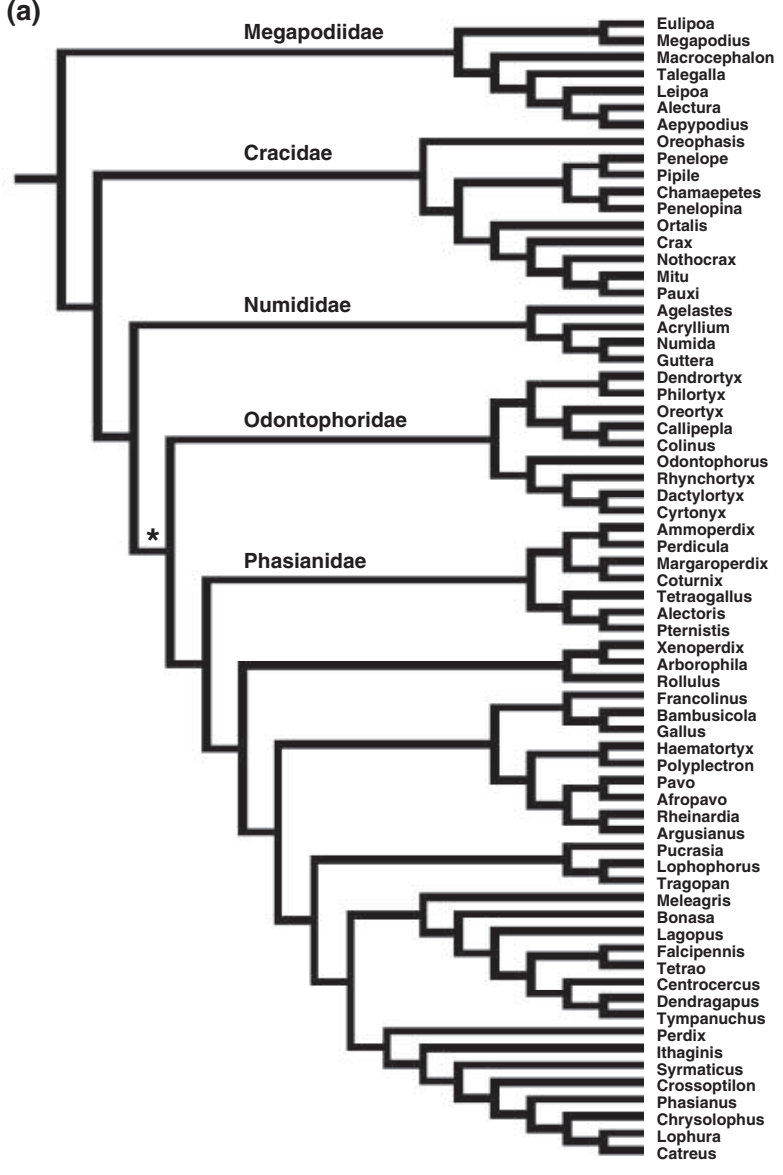

(b)

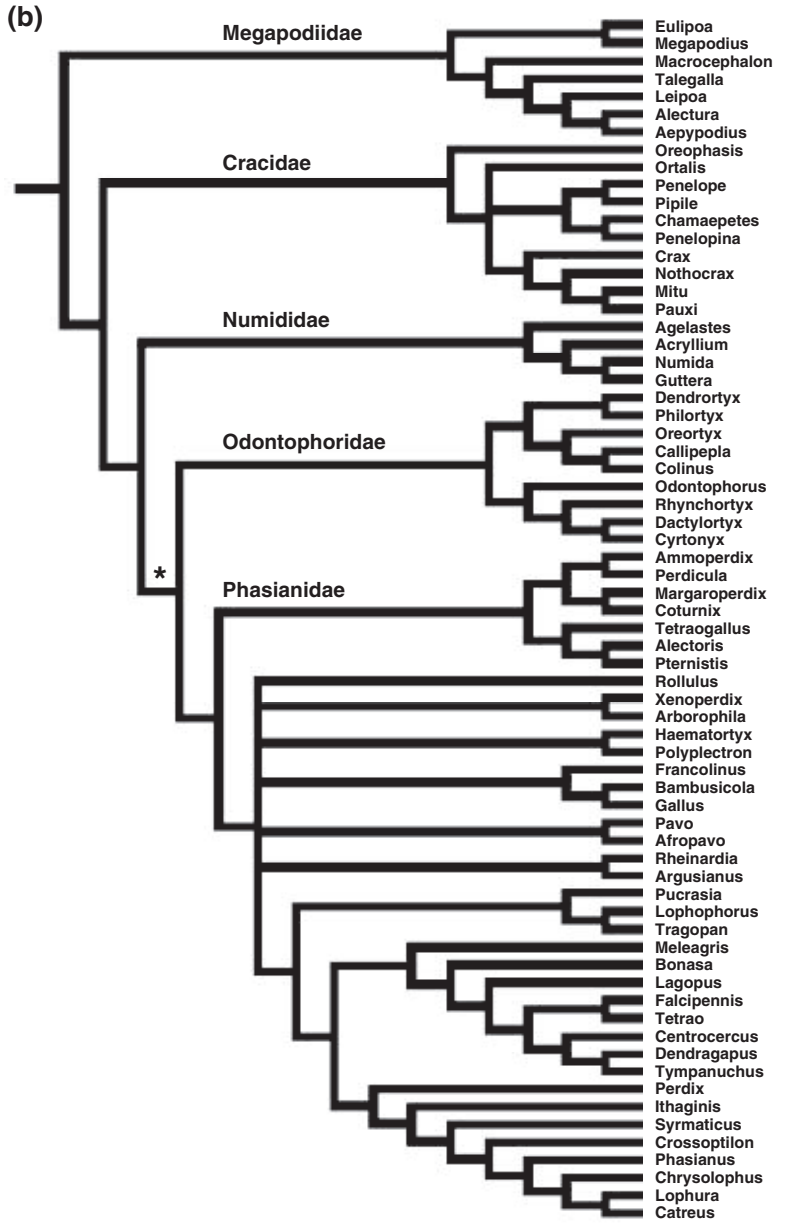

Fig. 1 Genera-level supertrees for the Galliform birds based on additive binary coding of source trees: fully resolved 'best informed guess' topology (a), composite supertree phylogeny (b), and 50\% majority rule consensus supertree (c). Each of the three topologies was also resolved so that the Odontophoridae was sister to the Numididae and the Phasianidae, by switching the placements of the Odontophoridae and the Numididae at the node marked with an asterisk. Figure 1 continued.

(see Results) regardless of which tree was used, we only report the results from the phylogenetic analyses based on the BIG tree. For the analysis of the relationship between SSD and MS, the low sample size did not allow for a phylogenetically independent matched pairs analysis (see Harvey \& Pagel, 1991) as it only yielded very few matched pairs. Instead, we used a normal ANCOVA with SSD as the dependent continuous variable and MS (monogamy or polygamy) as a categorical independent variable and mean body mass (sexes pooled) as a covariate for the raw data at the genus level. This allowed us to estimate the relationship between these variables while controlling for body mass. All data were $\log _{10}$ transformed prior to raw data analyses and before calculations of independent contrasts to ensure normality. Independent contrasts were calculated using the PDAP: PDTREe module within Mesquite (Midford et al., 2002; Maddison 8 Maddison, 2004).
For the analyses of directional evolution of SSD in relation to female life history traits, we used DISCRETE (4.0) (Pagel, 1994, 1997). This program is based on a Markov model for trait evolution and allows for estimation of ancestral states, investigation of correlated evolution between two traits, investigation of the directionality of changes in traits, and how changes in one trait precedes changes in another trait. A likelihood ratio test is used to compare the maximum likelihood fits of a model that only allows for independent evolution of two traits to a model that allows for dependent evolution between two traits. The likelihood ratio test statistic is $\chi^{2}$ distributed with d.f. $=4$ for the comparison between the independent and the dependent model (Pagel, 1997).

One can investigate the pattern of co-evolution for a pair of traits through investigation of the relative magnitudes of their joint transition rates (see Fig. 2). 


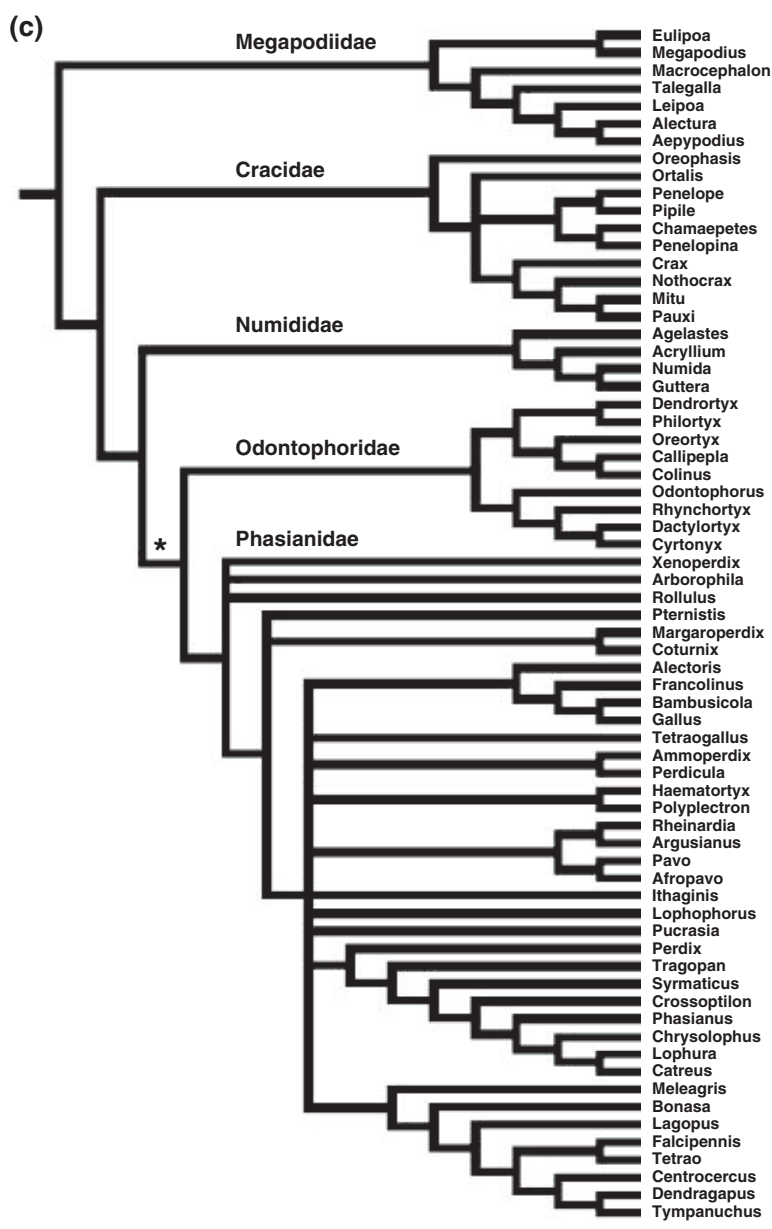

Fig. 1 Continued.
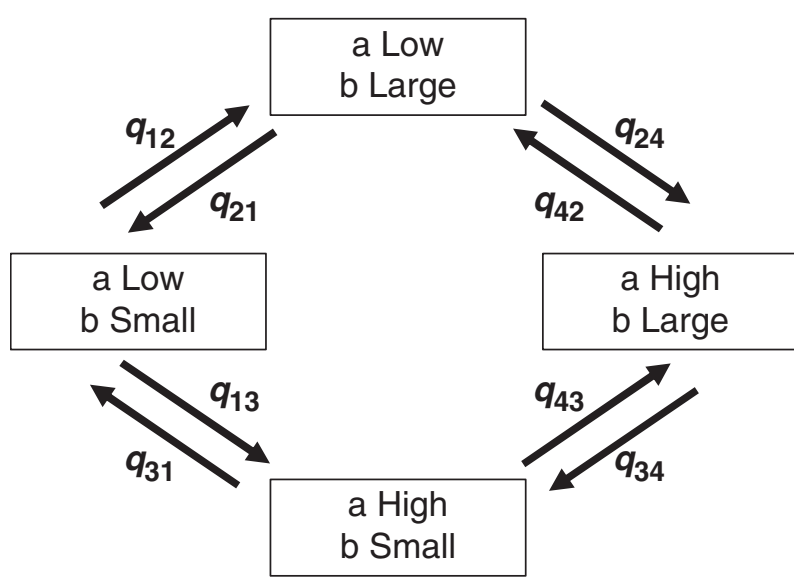

Fig. 2 Flow diagram of the possible transitions of a hypothetical model of dependent correlated evolution of two traits ( $a$ and $b$ ) that can take two stages each (low or high; small or large). Each potential transition is given by $q_{\mathrm{ab}}$.
So, for example, one can ask which path (upper vs. lower) from 'low-small' to 'high-large' is most likely on the data. One approach (see e.g. Cézilly et al., 2000; Kolm et al., 2006) is to ask which of the eight joint rates (represented by the arrows in Fig. 2) are indistinguishable from zero, using a likelihood ratio test of the nested models (focal rate $=0$ ) vs. (focal rate $=M L$ estimate). Rates that are indistinguishable from zero suggest that these paths are unlikely. If one can identify the ancestral states (i.e. which box in Fig. 2 is ancestral), a full description of the likely evolutionary paths through time is possible.

Following transformation of female body mass, egg size and clutch size into discrete characters, body mass and egg size showed a perfect relationship (i.e. for the 34 genera for which we had data on these traits, all genera with larger than average females had larger than average eggs and vice versa). As Discrete assumes no simultaneous changes in two traits, we could thus not disentangle the directional evolution of these two traits in relation to each other. Moreover, this perfect correlation also meant that we only performed a discrete analysis on SSD in relation to female body mass. As the bivariate contrast analyses did not show any relationship between female body mass and clutch size, we did not perform any DISCRETE analysis for this combination of traits. Further, although it would be very interesting to perform a DISCRETE analysis on clutch size in relation to egg size to disentangle the evolution of these two traits for Galliformes (as done for cichlid fishes by Kolm et al., 2006), DISCRETE requires larger sample sizes ( $\mathrm{N}$. Kolm, personal observation) than we had for robust tests. To investigate whether the relative frequency of trait values might affect our results (Nosil \& Mooers, 2005) for our data, we randomized the distribution of female body mass and SSD (from Fig. 5) across one of our BIG trees 100 times and then performed directional DisCRETE analyses to investigate how often chance alone would yield the same result as from our DISCRETE analyses based on the actual transitions in the tree. Only one of our 100 randomized datasets produced the same significant set of transitions, suggesting this was not a problem.

\section{Results}

\section{Analyses based on raw data}

Genera with higher female body mass had larger eggs (Fig. 3a). There was a nonsignificant trend suggesting a negative relationship between female body mass and clutch size (Fig. 3b). Egg size was negatively correlated to clutch size (Fig. 3c), and this result was robust also when we controlled for female body mass using a multiple regression analysis (Multiple $r^{2}=0.91, F_{2,30}=148.1$, $P<0.0001$; partial $\left.r=-0.63, t_{30}=4.5, P=0.0001\right)$. Both female body mass and egg size were positively 
(a)

(c)

(a) Egg size correlation: $n=34, r=0.92, P=0.0001)$. (b) Clutch size vs. female body mass (Pearson correlation: $n=48, r=-0.26, P=0.077)$. (c) Egg size vs. clutch size (Pearson correlation: $n=38, r=-0.44, P=0.006$ ).

(d) Female body mass vs. sexual size dimorphism (SSD) (Pearson correlation: $n=53, r=0.38, P=0.005$ ). (e) Egg size vs. SSD (Pearson correlation: $n=34, r=0.49$, $P=0.004$ ). (f) Clutch size vs. SSD (Pearson correlation: $n=48, r=0.10, P=0.49)$.
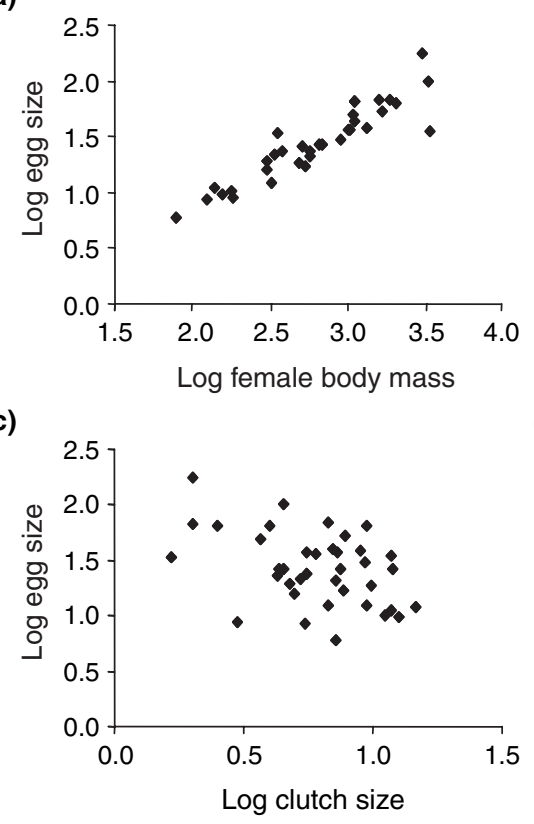

(b)

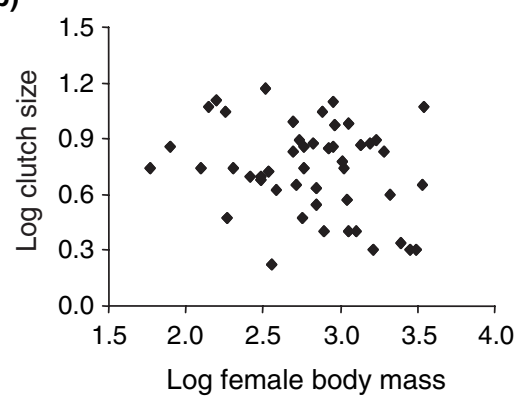

(d)

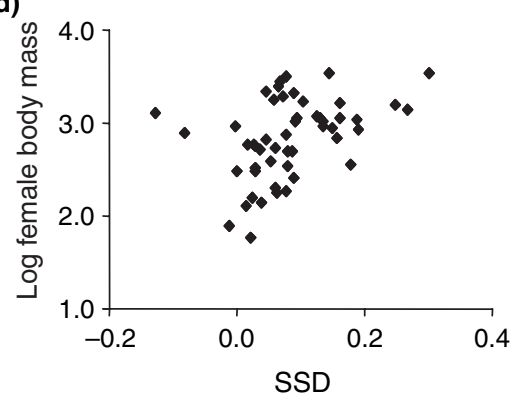

(f)

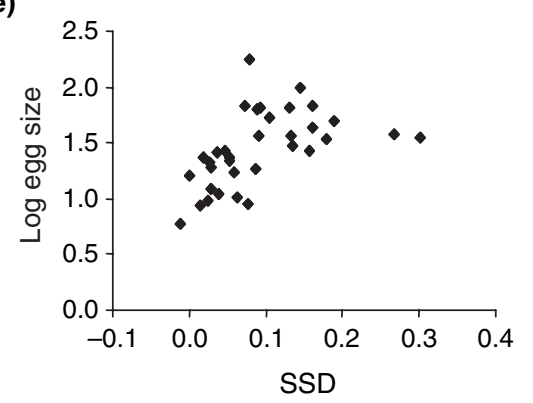

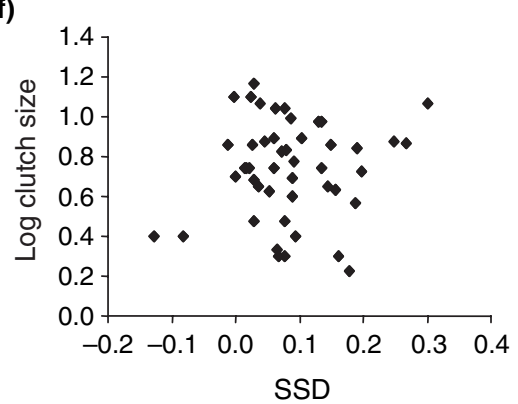

related to SSD (Fig. 3d,e). Clutch size was independent of SSD (Fig. 3f). Polygynous genera had higher levels of SSD than monogamous genera [polygynous genera (mean SSD $\pm \mathrm{SE}$ ): $0.15 \pm 0.02$; monogamous genera: $\left.0.06 \pm 0.02, F_{1,21}=10.3, P<0.01\right]$. This result remained statistically significant also when analysed using an ANCova with the mean body mass of males and females as a covariate $\left(F_{1,20}=6.0, P<0.05\right)$.

\section{Phylogenetic analyses}

The PIC analyses supported those on the raw data. Genera with higher female body mass had larger eggs and egg size was significantly negatively related to clutch size for all but one of the BIG trees (Fig. 4a,c). This negative relationship held consistently across analyses for all trees when controlling for female size in a multiple regression analysis (Multiple $r^{2}=0.87, F_{2,30}=104.9$, $P<0.0001$; partial $\left.r=-0.45, t_{30}=2.8, P<0.01\right)$. However, there was no significant relationship between female body mass and clutch size (Fig. 4b). Female body mass and egg size were positively related to SSD
(Fig. 4d,e) but again, there was no relationship between clutch size and SSD (Fig. 4f).

Few contemporary Galliform genera consisted of largebodied females with low SSD or small-bodied females with high SSD after these variables were transformed into binary characters (Fig. 5). The DISCRETE analysis of female body mass and SSD therefore confirmed the contrast analyses on the relationship between these variables. The dependent model provided a better fit than the independent model, which is consistent with correlated evolution of these two traits (the log-likelihood for the independent model was -64.1 compared with -58.1 for the dependent model, $L R=11.9$, $P<0.05)$. The 'local' ancestral state (Pagel, 1999) for female body mass could not be determined with certainty (high female body mass: 58\% posterior probability; low female body mass: $42 \%$ posterior probability; $L R=0.63$, d.f. $=1, P=0.43)$. The ancestral state for SSD could also not be determined with certainty although the trend suggested that the ancestral state was more likely to be low SSD (high SSD: $21 \%$ probability; low SSD: 79\%; $\mathrm{LR}=2.1$, d.f. $=1, P=0.13)$. 
(a)

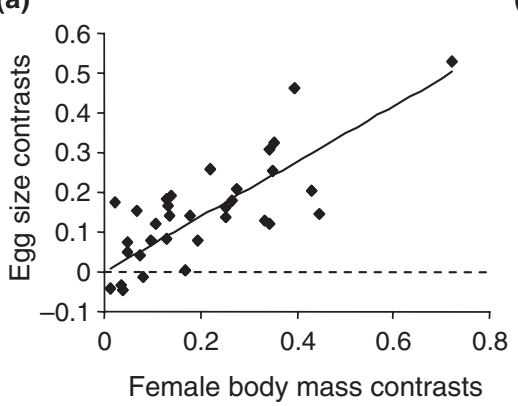

(c)

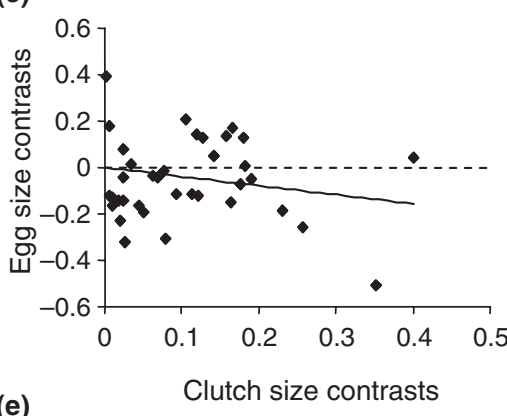

(e)

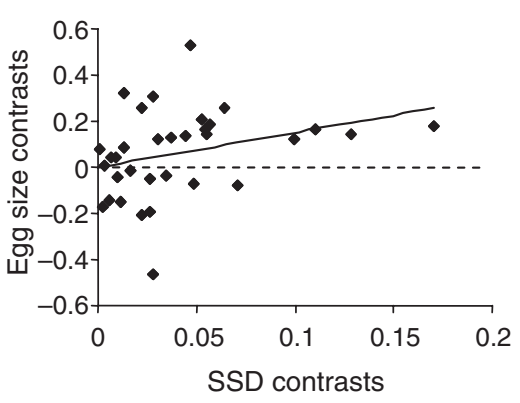

(b)

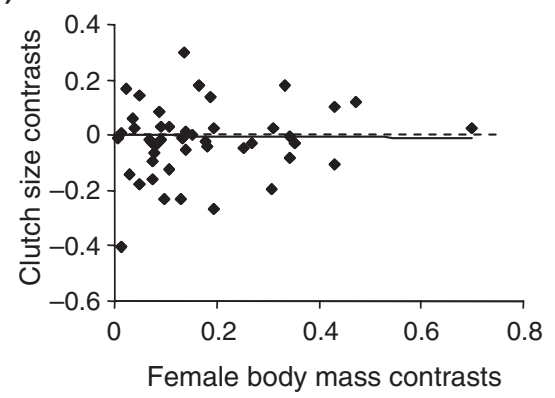

(d)

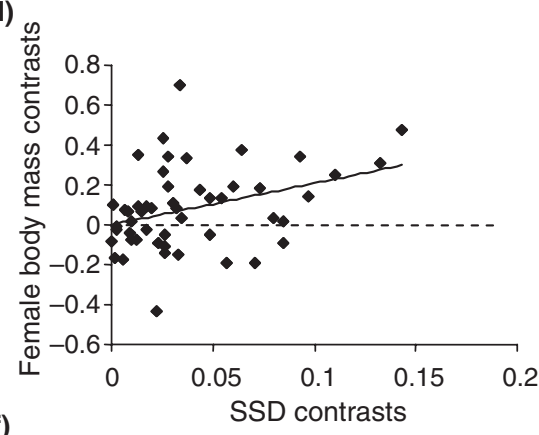

(f)

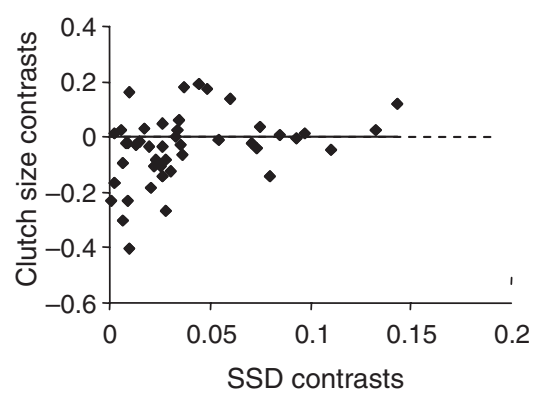

Fig. 4 Bivariate regressions based on independent contrasts and performed through the origin, with the independent variable positivized. The dashed line represents $y=0$. (a) Egg size vs. female body mass $\left(t_{32}=12.5\right.$, $r=0.91, P<0.0001$ ). (b) Clutch size vs. female body mass $\left(t_{45}=0.17, r=-0.03\right.$, $P=0.87)$. (c) Egg size vs. clutch size $\left(t_{36}=\right.$ 1.9, $r=-0.31, P=0.06$ (note that this negative relationship was statistically significant at $P<0.05$ for all other trees, see text for details)). (d) Female body mass vs. sexual size dimorphism (SSD) $\left(t_{49}=3.9, r=0.49\right.$, $P=0.0003)$. (e) Egg size vs. SSD $\left(t_{32}=2.7\right.$, $r=0.43, P=0.01)$. (f) Clutch size vs. SSD $\left(t_{45}=0.02, r=-0.003, P=0.98\right)$.
The evolutionary pathways between female body mass and SSD in relation to each other is presented in a flow diagram (Fig. 6). The results from this analysis show that the most likely path from small bodied females with low SSD towards a large body with high SSD is via the bottom path, such that SSD increases first. The only $P$-value that changed (i.e. from $P<0.05$ to $P>0.05$ or vice versa) depending on which tree was used across all analyses was for the transition between a large bodied female with high SSD towards a small bodied female with high SSD [i.e. transition $q_{34}$ (Fig. 6) changed from $P<0.05$ for all other trees to $P=0.10$ for one of the BIG trees]. However, the transition rate parameter for this transition was higher than the lowest significant transition rate parameter for any other transition also for this tree (see Kolm et al., 2006 for discussion on this). We therefore suggest that this transition is most likely to be common. In contrast, the most likely route for large bodied females with high SSD towards a small body with low SSD seems via an initial decrease in body mass. Hence, increases in female body size have followed after increases in the levels of sexual selection resulting in increased SSD whereas decreases in SSD have only followed after decreases in female body size. As all genera with large bodied females also lay large eggs and vice versa (see

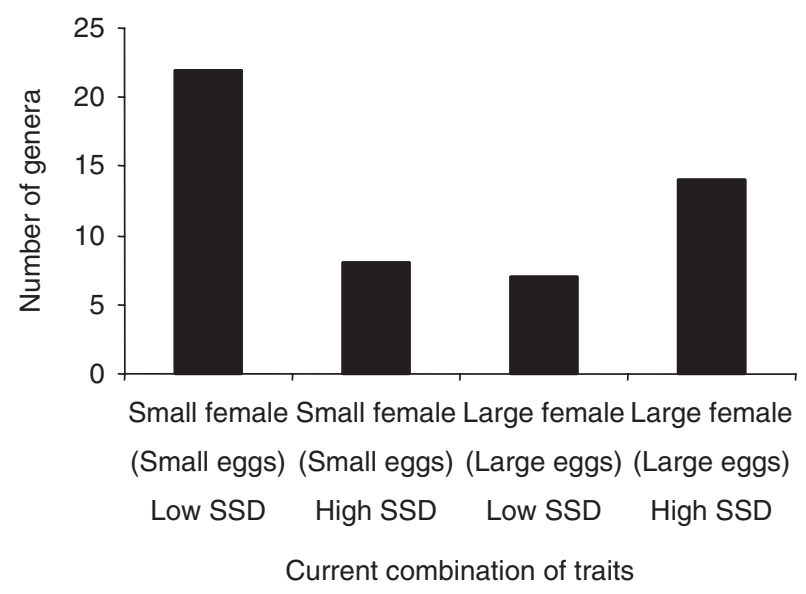

Fig. 5 Current combinations of states of Female body mass (= Egg size) and sexual size dimorphism Galliform genera. 


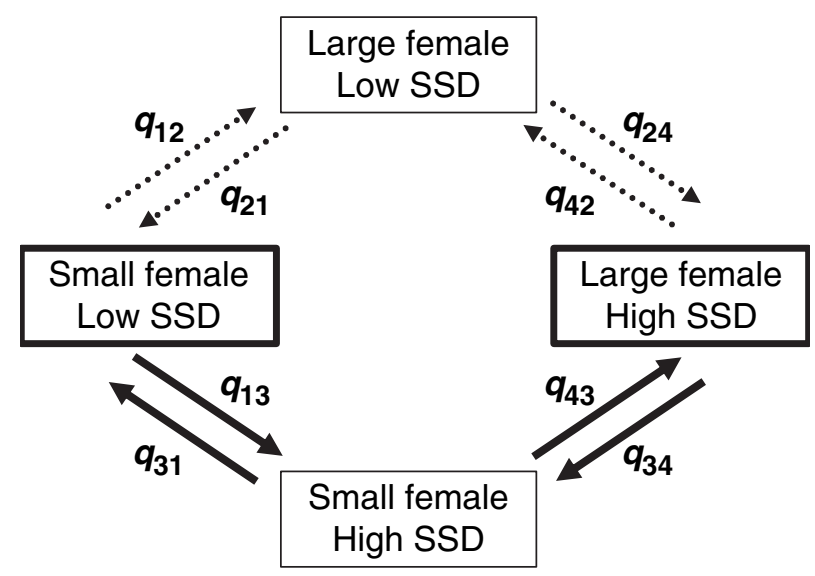

Fig. 6 Flow diagram over the evolutionary transitions between female body mass and sexual size dimorphism (SSD). The common current states are highlighted by boldly lined boxes. Significant transitions are marked by solid arrows whereas nonsignificant and thus unlikely transitions are marked by dotted arrows. Statistics for individual transitions are as follows $(n=51): q_{12}$ (transition rate parameter) $=0.05, L R=2.8, P=0.09 ; q_{21}=0.24, L R=1.5, P=$ $0.22 ; q_{24}=0.10, L R=2.2, P=0.14 ; q_{42}=0.00007, L R=0.06$, $P=1.0 ; q_{34}=0.46, L R=2.6, P=0.10$ (note that this transition rate parameter was high and also statistically significant across all other trees), $q_{43}=0.70, L R=4.8, P=0.03 ; q_{31}=0.96, L R=8.6$, $P=0.003 ; q_{13}=0.41, L R=5.8, P=0.02$. Note that a similar relationship would be obtained between egg size and SSD as all genera with high female body mass also laid large eggs and vice versa.

comment on this in the Methods), it means that the DISCRETE analyses would yield similar results if female body mass was exchanged with egg size.

\section{Discussion}

Our results showed that egg size is more strongly related to female body mass in Galliformes than is clutch size. Sexual selection was strongly linked to female body mass and egg size in this group and our DISCRETE analysis showed that increases in female body mass and egg size most likely occurred following initial increases in SSD. This suggests that sexual selection has been an important determinant in driving the evolution of these female traits and that these traits have not just been driven by a genetic correlation between male and female body size. Egg size and clutch size were negatively related suggesting a trade-off between these traits in this group.

Our analyses of the interactions among these female traits show, in agreement with many other studies on birds, a strong positive relationship between egg size and female size in Galliformes. Despite the generality of this pattern across taxa (e.g. Roff, 1992), the ultimate causes to this relationship are still poorly known. However, physiological limitations may hinder small species from evolving large eggs, as has been suggested in cichlid fishes (Kolm et al., 2006). Also, the egg to body size ratio is generally high in Galliformes, as might be expected in a group that produces precocial young, making them more likely to reach their physiological limit than many other avian groups.

Our analysis of SSD in relation to MS indicate that SSD indeed is a good measure of the level of sexual selection in this group, particularly as this result held also after controlling for female size (Björklund, 1990; Bennett \& Owens, 2002). Both female body mass and egg size were positively related to SSD in agreement with other studies (Weatherhead \& Teather, 1994; Figuerola \& Green, 2006). However, our DISCRETE analysis suggests that increases in sexual selection have preceded increases in female size. Although we could not establish the ancestral states of female body mass and SSD with certainty, we found a trend suggesting that the ancestral Galliform genus had a low level of SSD. Hence, as can be seen in Fig. 6, the most likely route from a small female body mass to a large female body mass (and small egg size to large egg size) is via an increase in SSD. However, the opposite route, from the combination of a large female with high SSD to that of a small female body with low SSD is also possible (Fig. 6) and indeed may often occur by changes in size preceding changes in SSD. These two routes are consistent with the idea that evolutionary transitions happen relatively freely between these combinations of states. Moreover, because our directional analysis suggests that because increases in female size have occurred after and not only at the same time as increases in SSD, female body mass likely does more than just co-vary with male size through a strong genetic correlation (e.g. Weatherhead \& Teather, 1994). Together with our results on the life history interactions, this points towards the Galliform genera having evolved along a continuum between two strategies: (1) genera with low levels of sexual selection, small females, large clutches and small eggs and (2) genera with high levels of sexual selection, large females, small clutches and large eggs. Our dataset is somewhat limited as it does not include data on the number of clutches that are produced per year. This additional variable would be beneficial to include in future studies. However, we believe that our description of the evolution along this continuum is at least indicative for Galliformes. Although it is well known that birds have evolved along a slow-fast continuum and that the positioning along this continuum is related to body size and egg size (fast: small body size, small eggs, fast reproduction; slow: large body size, large eggs, slow reproduction) (e.g. Roff, 1992, 2002 and references therein), we are not aware of anyone implicating sexual selection as an evolutionary driver affecting movement along this particular continuum.

Why might female body mass increase after increases in SSD if not only through a strong genetic correlation between female body mass and male body mass? Given 
the interaction between female size, egg size and clutch size in Galliformes, we cannot be certain whether it is female body size per se or positive selection for egg size (and/or negative selection for clutch size) that covaries with increases in SSD. For instance, increased levels of sexual selection in males often co-vary with decreases in male care in birds (e.g. Andersson, 1994 and references therein). Precocial chicks will need a larger egg investment and if smaller clutches of larger eggs require less care than larger clutches of small eggs, increases in SSD could well co-vary with changes in MS from monogamy to polygamy (as suggested by our analysis of SSD in relation to MS). Hence, for genera evolving from monogamy to polygamy, increasing body mass and egg size at the cost of clutch size could be a way for females to maximize offspring success when minimal paternal care is available. Future analyses on systems where data on paternal care is readily available could address how changes in these female traits may have occurred along changes in paternal care to test this hypothesis. An alternative explanation that we find interesting is that increases in SSD may lead to increases in female-female competition and hence selection for increased female size. This hypothesis was originally put forth by Langston et al. (1990) who suggested that a major cost of polygyny for females is competition for resources. Hence, increases in SSD (coupled to increases in the level of polygyny) may lead to increased competition for resources among females which in term selects for increased female size if larger females are better competitors (Langston et al., 1990). Supporting this, such a pattern has been found in red-winged blackbirds (Agelaius phoeniceus; Langston et al., 1990) and also in other groups of birds (e.g. dunnocks, Prunella modularis; Langmore et al., 2002).

Few studies have detected a negative relationship between egg size and clutch size in birds, which raises the question of why it is evident in Galliformes. Galliform birds are precocial with relatively little post-hatching parental care and the majority of a female's investment goes into eggs, which tend to be disproportionately large compared with those laid by altricial birds. Building on the arguments by Lack (1967), we suggest that this causes a constraint in resources which cannot be mediated at the parental care stage (as can occur in altricial species) leading to a clear trade-off between egg size and egg number in this group of birds. Supporting this, negative relationships between egg size and egg number in birds have in fact most often been found in precocial groups of birds (Lack, 1968; Blackburn, 1991a; Rohwer, 1991; Figuerola \& Green, 2006; this study; but see Blackburn, 1991b). Given the differences between different studies of different groups of birds, future broad scale analyses, using similar data sets to that of Bennett $\delta$ Owens (2002) or meta-analyses, would prove fruitful to identify the ultimate causes to why some, but not all, groups of birds show a trade-off between egg size and egg number (see also Blackburn, 1991b and Martin et al.,
2006, for discussion on this). Predation should also be considered more carefully.

To conclude, based on a new genus level supertree of Galliformes and DisCRETE analyses, our results suggest that sexual selection leading to higher levels of SSD has been an important driver of female body mass and egg size in this group. Moreover, our results show that these increases in female size are not simply by-products of selection for increased male size through a strong genetic correlation. We suggest that future studies should incorporate sexual selection to a much higher degree in order to fully understand the reasons for the extreme variation in female life histories among contemporary taxa.

\section{Acknowledgments}

N. K. was supported by a Short Visit Fellowship from The Royal Society and E. J. A. C. by a University Research Fellowship from The Royal Society. We thank Rutger Vos and Aki Mimoto for assistance with construction of the genera-level supertree and the $\mathrm{FAB}^{*} \mathrm{lab}$ at SFU for feedback on this work. We also thank John Reynolds for facilitating this collaboration.

\section{References}

Andersson, M. 1994. Sexual Selection. Princeton University Press, Princeton, New Jersey.

Armstrong, M.H., Braun E.L. \& Kimball T.T. 2001. Phylogenetic utility of avian ovomucoid intron G: a comparison of nuclear and mitochondrial phylogenies in Galliformes. Auk 118: 799804.

Baum, B.R. 1992. Combining trees as a way of combining data sets for phylogenetic inference, and the desirability of combining gene trees. Taxon 41: 1-10.

Bennett, P.M. \& Owens, I.P.F. 2002. Evolutionary Ecology of Birds. Oxford university Press, Oxford.

Bininda-Emonds, O.R.P., Jones, K.E., Price, S.A., Cardillo, M., Greyner, R. \& Purvis, A. 2004. Garbage in, garbage out: data issues in supertree construction. In: Phylogenetic Supertrees: Combining Information to Reveal the Tree of Life (O. R. P. BinindaEmonds, ed.), pp. 267-280. Kluwer Academic, Dordrecht, the Netherlands.

Birks, S.M. \& Edwards, S.V. 2002. A phylogeny of the megapodes (Aves: Megapodiidae) based on nuclear and mitochondrial DNA sequences. Mol. Phyl. Evol. 23: 408-421.

Björklund, M. 1990. A phylogenetic interpretation of sexual dimorphism in body size and ornament in relation to mating system in birds. J. Evol. Biol. 3: 171-183.

Blackburn, T.M. 1991a. The interspecific relationship between clutch size and egg size in wildfowl. Auk 108: 209-211.

Blackburn, T.M. 1991b. An interspecific relationship between egg size and clutch size in birds. Auk 108: 973-977.

Bloomer, P. \& Crowe, T.M. 1998. Francolin phylogenetics: molecular, morphobehavioral, and combined evidence. Mol. Phyl. Evol. 9: 236-254.

Bush, K.L. \& Strobeck, C. 2003. Phylogenetic relationships of the Phasianidae reveals possible non-pheasant taxa. Heredity $\mathbf{9 4 :}$ 472-489. 
Cézilly, F., Dubois, F. \& Pagel, M. 2000. Is mate fidelity related to site fidelity? A comparative analysis in Ciconiiforms. Anim. Behav. 59: 1143-1152.

Chubb, A.L. 2004. New nuclear evidence for the oldest divergence among neognath birds: the phylogenetic utility of ZENK (i). Mol. Phyl. Evol. 30: 140-151.

Cornishbowden, A. 1986. Nomenclature for Incompletely Specified Bases in Nucleic-Acid Sequences Recommendations 1984 - Nomenclature Committee of the International Union of Biochemistry (Nc-Iub). J. Biol. Chem. 261: 13-17.

Cramp, S. \& Simmons, K.E. 1980. The birds of the western Palearctic, Vol. 2. Oxford University Press, Oxford.

Crowe, T.M. 1978. The evolution of guinea-fowl (Galliformes, Phasianidae, Numidinae) taxonomy, phylogeny, speciation and biogeography. Ann. S. Afr. Mus. 76: 43-136.

Crowe, T.M., Bloomer, P., Randi, E., Lucchini, V., Kimball, R., Braun, E. \& Groth, J.G. 2006. Supra-generic cladistics of landfowl (Order Galliformes). Acta Zoo. Sinica 52 (suppl): 385-361.

Cunningham, E.J.A. \& Russell, A.F. 2000. Egg investment is influenced by male attractiveness in the mallard. Nature 404: $74-77$.

Diaz-Uriarte, R. \& Garland, T. 1998. Effect of branch length errors on the performance of phylogenetically independent contrasts. Syst. Biol. 47: 654-672.

Dimcheff, D.E., Drovetski, S.V. \& Mindell, D.P. 2002. Phylogeny of tetraoninae and other galliform birds using mitochondrial 12 S and ND2 genes. Mol. Phyl. Evol. 24: 203-215.

Drovetski, S.V. 2002. Molecular phylogeny of grouse: individual and combined performance of W-linked, autosomal, and mitochondrial loci. Syst. Biol. 51: 930-945.

Dunn, P.O., Whittingham, L.A. \& Pitcher, T.E. 2001. Mating systems, sperm competition and the evolution of sexual dimorphism in birds. Evolution 55: 161-175.

Dunning, J.B. Jr. 1993. CRC Handbook of Avian Body Masses. CRC Press, Boca Raton, Florida.

Ellsworth, D.L., Honeycutt, R.L. \& Silvy, N.J. 1996. Systematics of grouse and ptarmigan determined by nucleotide sequences of the mitochondrial cytochrome- $b$ gene. Auk 113: 811-822.

Fairbairn, D.J. 1997. Allometry for sexual size dimorphism: pattern and process in the coevolution of body size in males and females. Ann. Rev. Ecol. Syst. 28: 659-687.

Felsenstein, J. 1985. Phylogenies and the comparative method. Am. Nat. 125: 1-15.

Figuerola, J. \& Green, A.J. 2006. A comparative study of egg mass and clutch size in the Anseriformes. J. Ornithol. 147: 57-68.

Fumihito, A., Miyake, T., Takada, M., Ohno, S. \& Kondo, N. 1995. The genetic link between the Chinese bamboo partridge (Bambusicola thoracica) and the chicken and junglefowls of the genus Gallus. Proc. Nat. Acad. Sci. 92: 11053-11056.

Geffen, E. \& Yom-Tov, Y. 2001. Factors affecting the rates of intraspecific nest parasitism among Anseriformes and Galliformes. Anim. Behav. 62: 1027-1038.

Gutiérrez, R.J., Zink, R.M. \& Yang, S.Y. 1983. Genic variation, systematic, and biogeographic relationships of some galliform birds. Auk 100: 33-47.

Gutiérrez, R.J., Barrowclough, G.F. \& Groth, J.G. 2000. A classification of the grouse (Aves: Tetraoninae) based on mitochondrial DNA sequences. Wildl. Biol. 6: 205-211.

Harvey, P.H. \& Pagel, M.D. 1991. The Comparative Method in Evolutionary Biology. Oxford University Press, Oxford, England.

Heath, D.D. \& Blouw, D.M. 1998. Are maternal effects in fishes adaptive or merely physiological side effects? In: Maternal
Effects as Adaptations (T. A. Mousseau \& C. W. Fox eds), pp. 178-210. Oxford University Press, Oxford, England.

Helm-Bychowski, K.M. \& Wilson, A.C. 1986. Raes of nuclear DNA evolution in pheasant-like birds: evidence from restriction maps. Proc. Nat. Acad. Sci. 83: 688-692.

Higgins, D.G., Blackshields, G. \& Wallace, I.M. 2005. Mind the gaps: progress in progressive alignment. Proc. Natl. Acad. Sci. 102: 10411-10412.

Holman, H.J. 1961. Osteology of living and fossil new world quails (Aves, Galliformes). Bull. Flor. St. Mus. 6: 131-233.

del Hoyo, J., Elliott, A. \& Sargatal, J. 1994. Handbook of the Birds of the World. Volume 2: New World Vultures to Guineafowl. Lynx Edicions, Barcelona.

Hoyt, D.F. 1979. Practical methods of estimating volume and fresh weight of bird eggs. Auk 96: 73-77.

Huelsenbeck, J.P. \& Ronquist, F. 2001. Mrbayes: Bayesian inference of phylogenetic trees. Bioinformatics 17: 754-755.

Kimball, R.T., Braun, E.L. \& Ligon, J.D. 1997. Resolution of the phylogenetic position of the Congo peafowl, Afropavo congensis: a biogeographic and evolutionary enigma. Proc. R. Soc. Lond. B 264: 1517-1523.

Kimball, R.T., Braun, E.L., Zwartjes, P.W., Crowe, T.M. \& Ligon, J.D. 1999. A molecular phylogeny of the pheasants and partridges suggests that these lineages are not monophyletic. Mol. Phyl. Evol. 11: 38-54.

Kimball, R.T., Braun, E.L., Ligon, J.D., Lucchini, V. \& Randi, E. 2001. A molecular phylogeny of the peacock-pheasants (Galliformes: Polyplectron spp.) indicates loss and reduction or ornamental traits and display behaviours. Biol. J. Linn. Soc. 73 : 187-198.

Kolm, N. 2001. Females produce larger eggs for large males in a paternal mouthbrooding fish. Proc. R. Soc. Lond. B 268: 22292234.

Kolm, N. \& Ahnesjö, I. 2005. Do egg size and parental care coevolve in fishes? J. Fish. Biol. 66: 1499-1515.

Kolm, N., Goodwin, N.B., Balshine, S. \& Reynolds, J.D. 2006. Life history evolution in cichlids 2: directional evolution of the trade-off between egg number and egg size. J. Evol. Biol. 19: $76-84$.

Kornegay, J.R., Kocher, T.D., Williams, L.A. \& Williams, A.C. 1993. Pathways of lysozyme evolution inferred from the sequences of cytochrome $b$ in birds. J. Mol. Evol. 37: 367-379.

Krüger, O. \& Davies, N.B. 2002. The evolution of cuckoo parasitism: a comparative analysis. Proc. R. Soc. Lond. B 269: 375-381.

Lack, D. 1967. The significance of clutch size in waterfowl. Wildfowl 19: 67-69.

Lack, D. 1968. Ecological Adaptations for Breeding in Birds. Methuen \& Co., London.

Langmore, N.E., Cockrem, J.F. \& Candy, E.J. 2002. Competition for male reproductive investment elevates testosterone levels in female dunnocks, Prunella modularis. Proc. R. Soc. Lond. $B$ 269: 2473-2478.

Langston, N.E., Freeman, S., Rohwer, S. \& Gori, D. 1990. The evolution of female body size in red-winged blackbirds: the effects of timing of breeding, social competition, and reproductive energetics. Evolution 44: 1764-1779.

Lindenfors, P., Székely, T. \& Reynolds, J.D. 2003. Directional changes in sexual size dimorphism in shorebirds, gulls and alcids. J. Evol. Biol. 16: 930-938.

Loytynoja, A. \& Goldman, N. 2005. An algorithm for progressive multiple alignment of sequences with insertions. Proc. Natl. Acad. Sci. 102: 10557-10562. 
Lucchini, V., Höglund, J., Klaus, S., Swenson, J. \& Randi, E. 2001. Historical biogeography and a mitochondrial DNA phylogeny of grouse and ptarmigan. Mol. Phyl. Evol. 20: 149-162.

Maddison, W.P. \& Maddison, D.R. 2004. Mesquite 1.01: A Modular System for Evolutionary Analysis. http://mesquiteproject.org/ mesquite/mesquite.html.

Martin, T.E., Bassar, R.D., Bassar, S.K., Fontaine, J.J., Lloyd, P., Mathewson, H.A., Niklison, A.M. \& Chalfoun, A. 2006. Life history and ecological correlates of geographic variation in egg and clutch mass among passerine species. Evolution 60, 390398.

Midford, P.E., Garland, T. Jr \& Maddison, W. 2002. PDAP: PDTREe package for Mesquite, version 1.01. http://mesquiteproject.org/pdap_mesquite/index.html.

Munechika, I., Nozawa, K. \& Suzuki, H. 1999. Relationships of Syrmaticus and Phasianus by Cyt-b gene array comparison. Jap. J. Ornithol. 47: 133-138.

Nishibori, M., Hayashi, T. \& Yasue, H. 2004. Complete nucleotide sequence of Numida meleagris (helmeted guineafowl) mitochondrial genome. J. Poultry. Sci 41: 259-268.

Nixon, K.C. 1999. The parsimony ratchet, a new method for rapid parsimony analysis. Cladistics 15: 407-414.

Nosil, P. \& Mooers, A.O. 2005. Testing hypotheses about ecological specialization using phylogenetic trees. Evolution 59: 2256-2263.

Nylander, J.A.A. 2004. mRmodeltest v2. Program distributed by the author. Evolutionary Biology Centre, Uppsala University, Uppsala.

Owens, I.P.F. \& Hartley, I.R. 1998. Sexual dimorphism in birds: why are there so many forms of dimorphism? Proc. $R$. Soc. Lond. B 265: 397-407.

Pagel, M. 1994. Detecting correlated evolution on phylogenies: a general method for the comparative analysis of discrete characters. Proc. R. Soc. Lond. B 255: 37-45.

Pagel, M. 1997. Inferring evolutionary processes from phylogenies. Zool. Scr. 26: 331-348.

Pagel, M. 1999. The maximum likelihood approach to reconstructing ancestral character states of discrete characters on phylogenies. Syst. Biol. 48: 612-622.

Pereira, S.L. \& Baker, A.J. 2004. Vicariant speciation of curassows (Aves, Cracidae): a hypothesis based on mitochondrial DNA phylogeny. Auk 121: 682-694.

Pereira, S.L., Baker, A.J. \& Wajntal, A. 2002. Combined nuclear and mitochondrial DNA sequences resolve generic relationships within the Cracidae (Galliformes, Aves). Syst. Biol. 51: 946-958.

Poiani, A. \& Jermiin L.S. 1994. A comparative analysis of some life-history traits between cooperatively and non-cooperatively breeding Australian passerines. Evol. Ecol. 8: 471-482.

Purvis, A. 1995. A modification to Baum and Ragan's method for combining phylogenetic trees. Syst. Biol. 44: 251-255.

Ragan, M.A. 1992. Phylogenetic inference based on matrix representation of trees. Mol. Phyl. Evol. 1: 53-58.

Randi, E., Fusco, G., Lorenzini, R. \& Crowe, T.M. 1991. Phylogenetic relationships and rates of allozyme evolution within the Phasianidae. Biochem. Syst. Ecol. 19: 213-221.

Roff, D.A. 1992. The Evolution of Life Histories: Theory and Analysis. Chapman \& Hall, New York, USA.

Roff, D.A. 2002. Life History Evolution. Sinauer Associates, Massachusetts, USA.

Rohwer, F.C. 1988. Inter- and intraspecific relationships between egg size and clutch size in waterfowl. Auk 105: 161-176.
Rohwer, F.C. 1991. Response to T. M. Blackburn. Auk 108: $211-$ 213.

Rolland, C., Danchin, E. \& de Fraipont, M. 1998. The evolution of coloniality in birds in relation to food, habitat, predation, and life-history traits: a comparative analysis. Am. Nat. 151: 514-529.

Ronquist, F. \& Huelsenbeck, J.P. 2003. mrbayes 3: Bayesian phylogenetic inference under mixed models. Bioinformatics 19: 1572-1574.

Saether, B.E. 1987. The influence of body weight on the covariation between reproductive traits in European birds. Oikos 48: 79-88.

Sheldon, B.C. 2000. Differential allocation: tests, mechanisms and implications. Trends Ecol. Evol. 15: 397-402.

Shibusawa, M., Nishibori, M., Nishida-Umehara, C., Tsudzuki, M., Masabanda, J., Griffin, D.K. \& Matsuda, Y. 2004. Karyotypic evolution in the Galliformes: an examination of the process of karyotopic evolution by comparison of the molecular cytogenetic findings with the molecular phylogeny. Cytogen. Gen. Res. 106: 111-119.

Sibley, C.G. \& Ahlquist, J.E. 1990. Phylogeny and Classification of Birds. Yale University Press, New Haven, Connecticut.

Sikes, D.S. \& Lewis, P.O. 2001. Beta Software, Version 1. PAuprat: PAUP* Implementation of the Parsimony Ratchet. Distributed by the Authors. Department of Ecology and Evolution, University of Connecticut, Storrs, USA.

Sorenson, M.D., Oneal, E., García-Moreno, J. \& Mindell, D.P. 2003. More taxa, more characters: the hoatzin problem is still unresolved. Mol. Biol. Evol. 20: 1484-1499.

Stearns, S.C. 1992. The Evolution of Life Histories. Oxford University Press, Oxford, England.

Swofford, D.L. 2002. PAUP*: Phylogenetic Analysis using Parsimony (* and Other Methods), Version 4. Sinauer Associates, Sunderland, MA.

Thompson, J.D., Gibson, T.J., Plewniak, F., Jeanmougin, F. \& Higgins, D.G. 1997. The CLUSTAL_X windows interface: flexible strategies for multiple sequence alignment aided by quality analysis tools. Nucleic Acids Res. 25: 4876-4882.

Thorely, J.L. \& Page, R.D.M. 2000. RADCon: phylogenetic tree comparison and concensus. Bioinformatics 16: 486-487.

Weatherhead, P.J. \& Teather, K.L. 1994. Sexual size dimorphism and egg-size allometry in birds. Evolution 48: 671-678.

Wen, L.-Y., Zhang, L.-X. \& Liu, N.-F. 2005. Phylogenetic relationship of Perdix dauuricae inferred from mitochondrial cytochrome $b$ gene. Zool. Res. 26: 69-75.

Wilkinson, M., Cotton, J.A., Creevey, C., Eulenstein, O., Harris, S.R., Lapointe, F.-J., Levasseur, C., Mcinerney, J.O., Pisani, D. \& Thorely, J.L. 2005. The shape of supertrees to come: tree shape related properties of fourteen supertree methods. Syst. Biol. 54: 419-431.

Young, K.A. 2005. Life-history variation and allometry for sexual size dimorphism in Pacific salmon and trout. Proc. $R$. Soc. Lond. B 272: 167-172.

Zhan, X.-J., Zhang, Z.-W., Wu, A.-P. \& Tao, Y.-J. 2003. Phylogenetic relationships of monal pheasants Lophophorus inferred from sequences of mitochondrial cytochrome $b$ gene. Zool. Res. 24: 337-342.

Zink, R.M. \& Blackwell, R.C. 1998. Molecular systematics of the scaled quail complex (genus Callipepla). Auk 115: 394-403.

Received 13 March 2006; revised 22 June 2006; accepted 24 August 2006 